\title{
Lowering hepatic venous pressure agent carvedilol versus variceal banding ligation for clinical outcomes of cirrhotic portal hypertension
}

This article was published in the following Dove Medical Press journal:

Therapeutics and Clinical Risk Management

\author{
Zhen-gang Wei ${ }^{1-3}$ \\ Feng-xian Wei ${ }^{1-3}$ \\ Zi-wei Shaol-3 \\ Guo-hong Su ${ }^{1-3}$ \\ Xue-ping $\mathrm{Qi}^{3}$ \\ You-cheng Zhangl-3 \\ 'Department of General Surgery, \\ Lanzhou University Second Hospital, \\ Lanzhou 730030, China; ${ }^{2}$ Hepato- \\ Biliary-Pancreatic Institute, Lanzhou \\ University Second Hospital, Lanzhou \\ 730030, China; ${ }^{3}$ Lanzhou University \\ Second Clinical Medical College, \\ Lanzhou University Second Hospital, \\ Lanzhou University, Lanzhou \\ 730030, China
}

Objective: Carvedilol is nonselective beta-blocker with a mild anti-alpha-1-adrenergic effect. Several studies proposed improved hemodynamic effects of carvedilol compared with propanolol. Our study was to perform a systematic review and meta-analysis of randomized control trials comparing carvedilol with variceal banding ligation (VBL).

Methods: Studies were searched on online databases MEDLINE, EMBASE(Ovid), the Cochrane Library, Chinese Wanfang Database, and China National Knowledge Infrastructure between January 2000 and May 2018. Incidence of bleeding and mortality were main outcome measures. Subgroup analysis and sensitivity analysis were conducted to ensure the robustness of pooled estimates.

Results: Ten randomized control trials including 1,269 cirrhotic patients were chosen. Compared with VBL, carvedilol showed similar preventive efficacy of risk ratios (RRs) in variceal bleeding, and bleeding-related mortality over different follow-up periods from 6 months to 24 months. Also, significant differences between carvedilol and VBL in overall mortality and other causes of mortality were failed to be found. Carvedilol achieved a lower incidence of portal hypertension gastropathy in both 6 months $(\mathrm{RR}=0.49,95 \% \mathrm{CI}: 0.38-0.64, P<0.00001)$ and 12 months ( $\mathrm{RR}=0.35,95 \% \mathrm{CI}: 0.26-0.47, P<0.00001)$. Two trials compared combination of carvedilol and VBL with VBL alone; however, the results failed to find an improved preventive efficacy of bleeding (RR=0.71, 95\% CI: $0.15-3.30, P=0.67$ ).

Conclusion: Carvedilol is equivalent to invasive VBL for variceal bleeding prevention. It can be well tolerated and may be of benefit to portal hypertension gastropathy. However, available data during 24 months follow-up did not support a potential advantage of carvedilol for prognosis as a lowering hepatic venous pressure agent.

Keywords: carvedilol, endoscopic ligation, VBL, variceal bleeding, cirrhosis, mortality

\section{Introduction}

Viral and alcoholic cirrhosis are common worldwide. Portal hypertension is its unavoidable complication in natural history, ${ }^{1}$ and variceal bleeding is its devastating consequence, ${ }^{2}$ bearing an incidence of $12 \%$, a recurrence of $60 \%$, and a mortality of $10 \%-15 \%$ in the first episode and $57 \%$ in the second episode. ${ }^{3,4}$ Treatment achieves hepatic venous pressure gradient (HVPG) reduction by $20 \%$ of baseline or immediate variceal eradication is effective to result in a significant reduction in the bleeding risk. ${ }^{5,6}$ Currently, nonselective beta-blockers (NSBBs) and endoscopic variceal banding ligation (VBL), which significantly reduce the bleeding risk, are recommended as first-line preventive therapy. ${ }^{7}$ Among kinds of NSBBs, carvedilol has a potential of decreasing intrahepatic resistance since its additional mild anti-alpha-1-adrenergic effect, and
Correspondence: You-cheng Zhang Department of General Surgery, Lanzhou University Second Hospital Cuiyingmen 82, Chengguan District, Lanzhou 730030, Gansu Province, Chin Tel +86 93I 5190524

Email zhangyouchengphd@I63.com 
have been reported to achieve a more HVPG reduction and higher hemodynamic response than propranolol. ${ }^{8}$

The HVPG is significantly associated with not only the risk of variceal bleeding, but also liver decompensation and hepatocellular carcinoma. ${ }^{9}$ A reduction in HVPG in cirrhotic patients may lead to an improved prognosis. ${ }^{10}$ Several studies have compared the medium and long-term outcomes of carvedilol with VBL yielding different results. ${ }^{11-20}$

The aim of the current review was to assess the potential of HVPG reduction of carvedilol compared with VBL for cirrhotic portal hypertension. With this aim, we conducted a meta-analysis and systematic review of randomized controlled trials (RCTs) comparing the influence of carvedilol with VBL on both variceal bleeding and long-term mortality.

\section{Methods}

\section{Search strategy and study selection}

This study was performed by following the guidelines of PRISMA. ${ }^{21}$ Records were identified through searching online databases including MEDLINE, EMBASE(Ovid), the Cochrane Library, Chinese Wanfang Database, and China National Knowledge Infrastructure Database. Additional records were identified through searching Cochrane control trials register center, manually screening reference, meeting abstracts, related articles and citations of searched studies, and also scholar website to enlarge the search results. The databases were searched from January 2000 to May 2018 using the following terms: carvedilol, beta-blocker, pharmacologic, endoscopic banding, band ligation, variceal bleeding, variceal hemorrhage, portal hypertension, and randomized trials. There was no limitation in language.

First, duplicates and studies without randomly designed grouping were excluded. After that, two reviewers screened the remained records, on the topic of carvedilol vs band ligation for variceal bleeding prevention dependently, based on detailed information of four items: participants, intervention, comparison, and outcome measures as listed in the following subtitles. For potential studies, both their abstracts and fulltexts were evaluated for eligibility. Final decisions of which study to include were made by the two reviewers through cross-checking and discussing their evaluation results. All of study information on authors, institute, and positive/negative study results was not blinded.

\section{Participants and intervention}

Participants in the included studies were cirrhosis patients with significantly portal hypertension symptoms as prevalence of esophageal varices, which is associated with risk of bleeding. Cirrhosis was diagnosed mainly based on clinical, radiological, biochemical items, and liver biopsy when available. Esophageal varices were under surveillance by follow-up endoscopy.

Oral carvedilol was compared with endoscopic varices banding ligation for variceal bleeding prevention. In treatment group, carvedilol was administered at a start dose of $6.25 \mathrm{mg}$ and was finally increased to $12.5 \mathrm{mg}$. The dose was aimed to be well tolerated without symptomatic hypertension and serious heart rate reduction. For comparison, VBL was performed every 2-4 weeks until esophageal varices became either grade I or complete obliteration. Other treatments among the groups were comparable.

\section{Data collection and outcome measures}

Data were extracted by another two independent reviewers and any discrepancies were resolved by discussion. In each trial, baseline information and outcome measures of interest were extracted: 1) baseline information - study country, case number, sex, average age, etiology (alcoholic, fatty liver disease, primary biliary cirrhosis, drug-induced injury, and others), and severity of cirrhosis (Child-Pugh score, model for end-stage liver disease score, and varices grade), other indexes reflecting liver function (creatinine, ascites, bilirubin, albumin, prothrombin time, portal vein congestion index, hyponatremia, and others), and follow-up periods; and 2) outcome measure - variceal bleeding rate, overall mortality, bleeding related and other causes of mortality, incidence of portal hypertensive gastropathy, and adverse events.

The primary outcome measure of prevention efficacy was variceal bleeding rate achieved by carvedilol and VBL during follow-up. Overall mortality and bleeding-related mortality were secondary outcome measures. Bleeding was considered as hematemesis and/or melena with endoscopic evidence of variceal bleeding, stigmata of recent hemorrhage with a $>2 \mathrm{~g} / \mathrm{dL}$ reduction in hemoglobin, and also included bleeding from banding ulceration. Bleeding-related mortality was defined as death within 6 weeks of the index bleed.

\section{Study quality assessment}

Two of the reviewers independently assessed the potential risk of bias due to inadequate methodological quality located in each trial. The Cochrane Handbook and the Cochrane tool of risk of bias assessment were adopted for assessing the quality of included randomized trials. ${ }^{22}$ Selection bias, performance bias, detection bias, attrition bias, reporting bias, and other bias were assessed by seven items: random 
sequence generation, allocation concealment, blinding of participants and personnel, blinding of outcome assessment, incomplete outcome data, selective reporting, and other possible bias. In each included trial, the quality was judged to be high when proper or adequate control of bias was mostly achieved; otherwise, the quality was judged to be inadequate or with unclear risk.

\section{Statistical analyses}

Data of outcome measures were reported as number of observation and/or proportion. Statistical analyses of data were performed by Revman 5.3 (The Cochrane Collaboration, Copenhagen, Denmark) in the fixed effects model if no significant inter-trials heterogeneity was found, or in the random effects model in case of significant heterogeneity. Heterogeneity was statistically assessed by chi-squared test with $I^{2}$ statistics and $P$-value expression. When $I^{2}>50 \%$ and $P<0.10$, the heterogeneity was considered significant.

The prevention efficacy was calculated as risk ratio (RR), which represented the relative risk of specific outcome incidence in the carvedilol group compared with that in VBL group. The pooled RR of outcome measure was reported as the summary statistic of prevention efficacy together with $95 \% \mathrm{CI}$ and $P$-value. Difference between groups were considered to be statistically significant when its $95 \% \mathrm{CI}$ not containing the value of 1 and its corresponding $P<0.05$.
For each analysis, the cause of heterogeneity was explored by reassessing risk of bias and handled by subgroup analysis. In current analysis, subgroup analysis was performed through: 1) bleeding and mortality measured time - 6, 12, 18, and 24 months; and 2) overall adverse event with different severity and detailed diagnosis. Stability of the results was explored by sensitivity analysis. In the current analysis, sensitivity analysis was performed through: 1) changing the combined model;2) excluding the inadequate quality of trial; and 3) excluding the trial responsible for the significant heterogeneity.

\section{Results Description of the included randomized control trials (RCTs)}

Overall, 409 potential references were retrieved in the literature search. Three hundred and ninety-four references were excluded by screening the titles and abstracts. The remaining 15 references were fully evaluated, and finally 10 of them, including 7 full-text papers and 3 meeting abstracts, were included in the study (Figure 1).

The characteristics of the ten included trials are shown in Table 1. The case number ranged from 25 patients to 125 patients in each group between 2009 and 2017. The total case number in the combined study were 1,269 patients, with 635 patients in the carvedilol group and 634 in the VBL group.
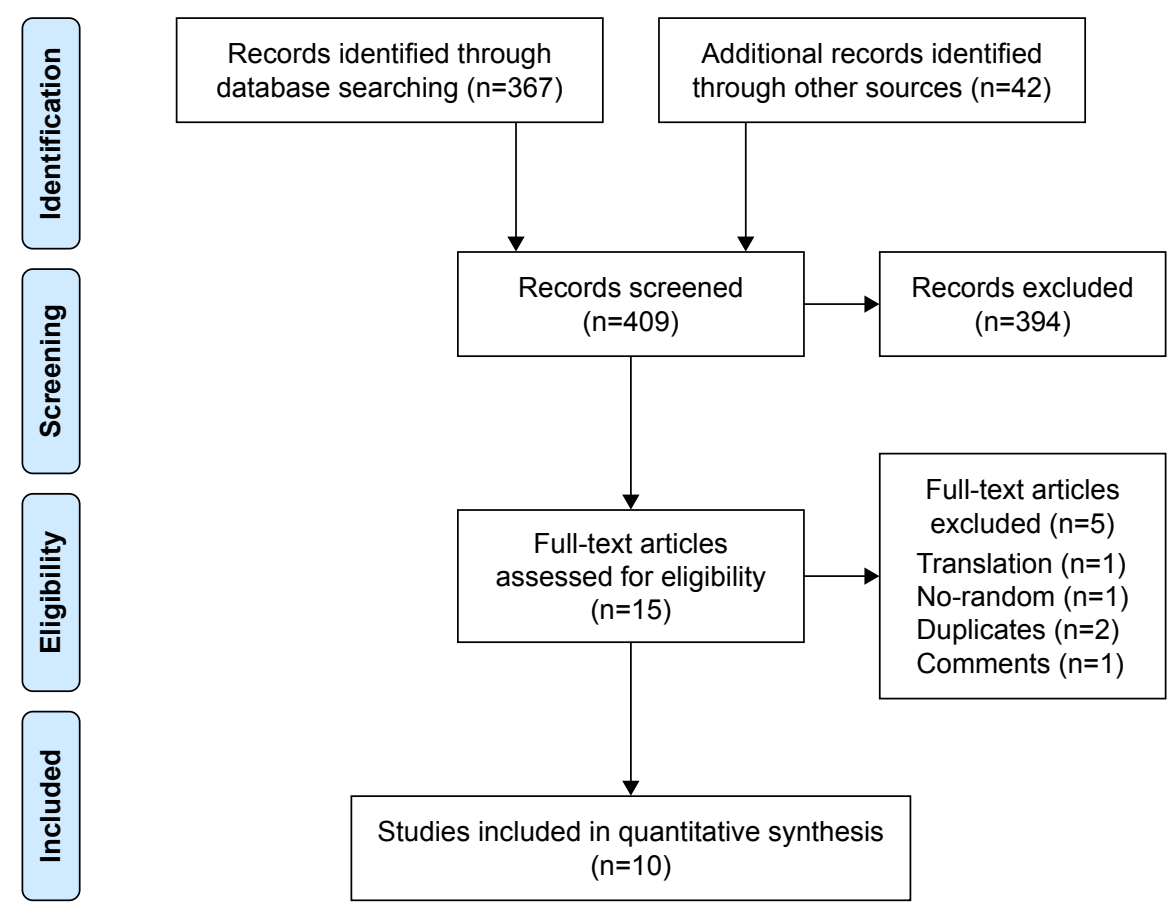

Figure I Flow diagram of the selection process of RCTs in the meta-analysis. Abbreviation: RCTs, randomized controlled trials. 


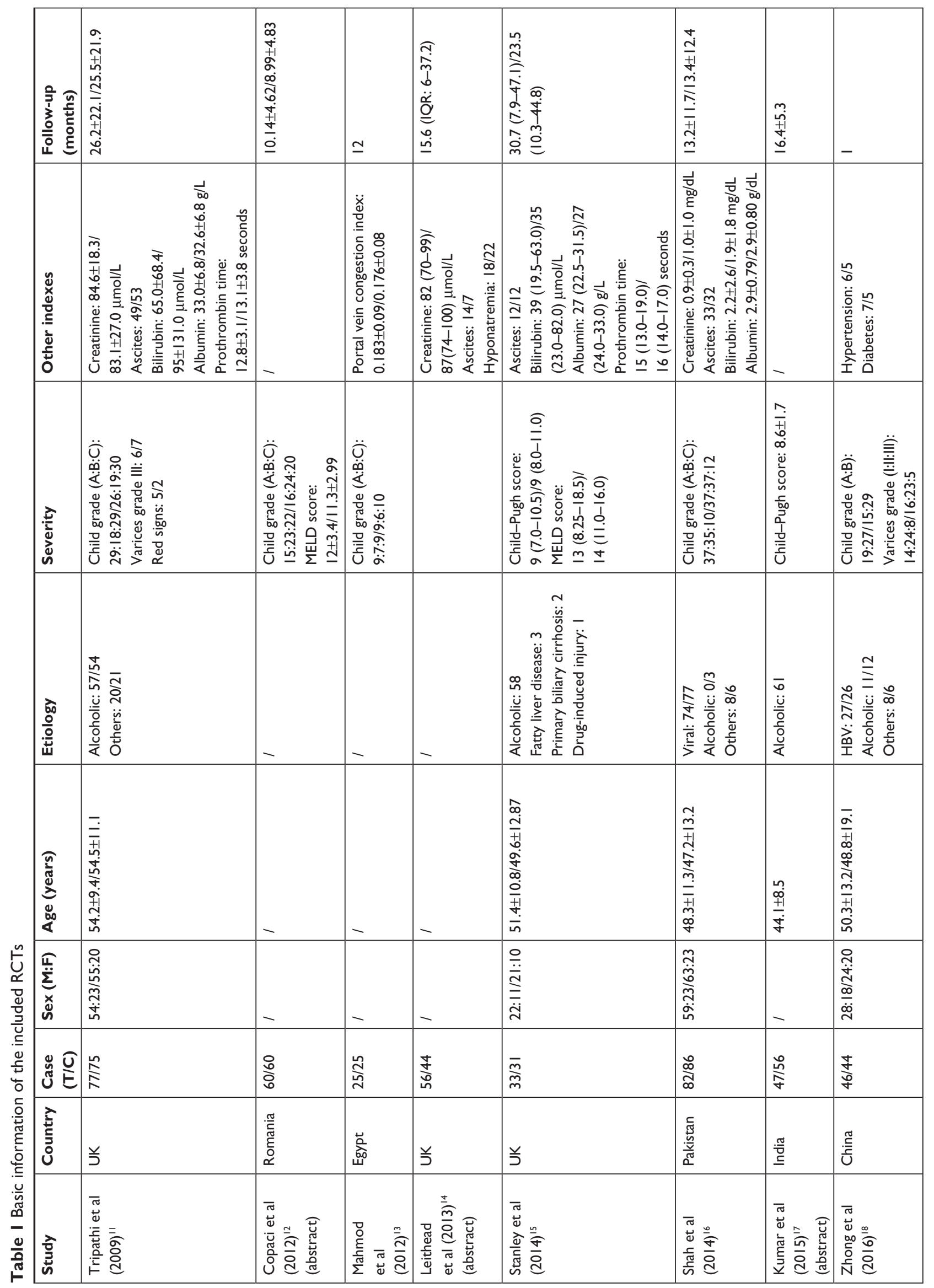




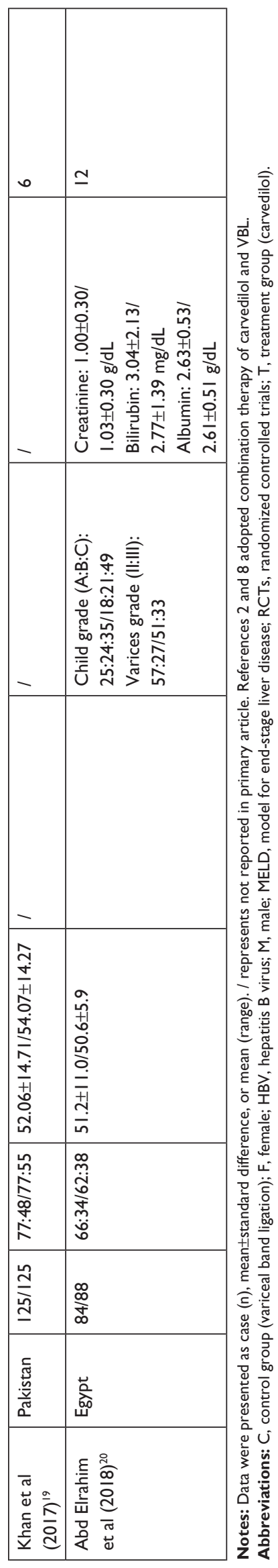

Sex composition and average age were comparable. Etiology of cirrhosis mainly included viral and alcoholic liver disease, and also fatty liver disease, primary biliary cirrhosis, and drug-induced injury. Severity of cirrhosis was measured according to the Child-Pugh score, Child grade, and model for end-stage liver disease score. Severity of varices was monitored by endoscopic varices grade and red sign. Other items indicating the function of liver included creatinine, ascites, bilirubin, albumin, and prothrombin time. Follow-up periods varied from 1 month to 26 months.

For methodological assessment, control of risk of bias was mainly inadequate in allocation concealment since five of them did not adopt a sealed opaque envelope method or center controlled randomization. Carvedilol was significantly different from VBL procedure, so blinding method in participants was impossible, only Shah et al adopted a center controlled grouping which may be helpful for blinding to personnel. Meanwhile, outcome measures were certainly incidence of bleeding, which would be hard influence by personnel willing, so majority of them were judged to be adequate. Two trials reported a combination therapy of carvedilol and VBL, ${ }^{12,18}$ which was different from the others in aspects of comparison, and they were judged high risk of bias in other bias. Overall quality of the included trials is low to moderate (Figure 2).

\section{Carvedilol versus VBL Variceal bleeding rate}

According to different outcomes measured time, we introduce a subgroup analysis first. In five trials, meta-analyses result showed that the pooled RR of variceal bleeding rate with the two groups was 0.71 in 6 months (95\% CI: $0.24-2.12, P=0.54$, $P^{2}=59 \%$ ), 0.69 in 12 months ( $95 \%$ CI: $0.36-1.30, P=0.25$, $P=0 \%$ ), 1.08 in 18 months ( $95 \% \mathrm{CI}: 0.63-1.86, P=0.78, P=0 \%$ ), and 0.87 in 24 months (95\% CI: $0.52-1.47, P=0.61, P^{2}=26 \%$ ). The results indicated that no significant difference was found in variceal bleeding rate between carvedilol and VBL group (Figure 3). Sensitivity analysis confirmed the principal analysis by changing combine model.

\section{Bleeding-related mortality}

Three trials reported data of bleeding-related mortality. Only one trial reported the data within 6 months, and showed no significant difference between carvedilol and VBL ( $\mathrm{RR}=1.95,95 \% \mathrm{CI}: 0.18-21.03, P=0.58$ ). Meta-analysis results showed similar results in 12 months $(\mathrm{RR}=1.15,95 \%$ CI: $\left.0.53-2.50, P=0.72, I^{2}=0 \%\right)$ and 24 months $(\mathrm{RR}=0.76$, $95 \%$ CI: $0.42-1.38, P=0.38, P^{2}=0 \%$ ). The results indicated 


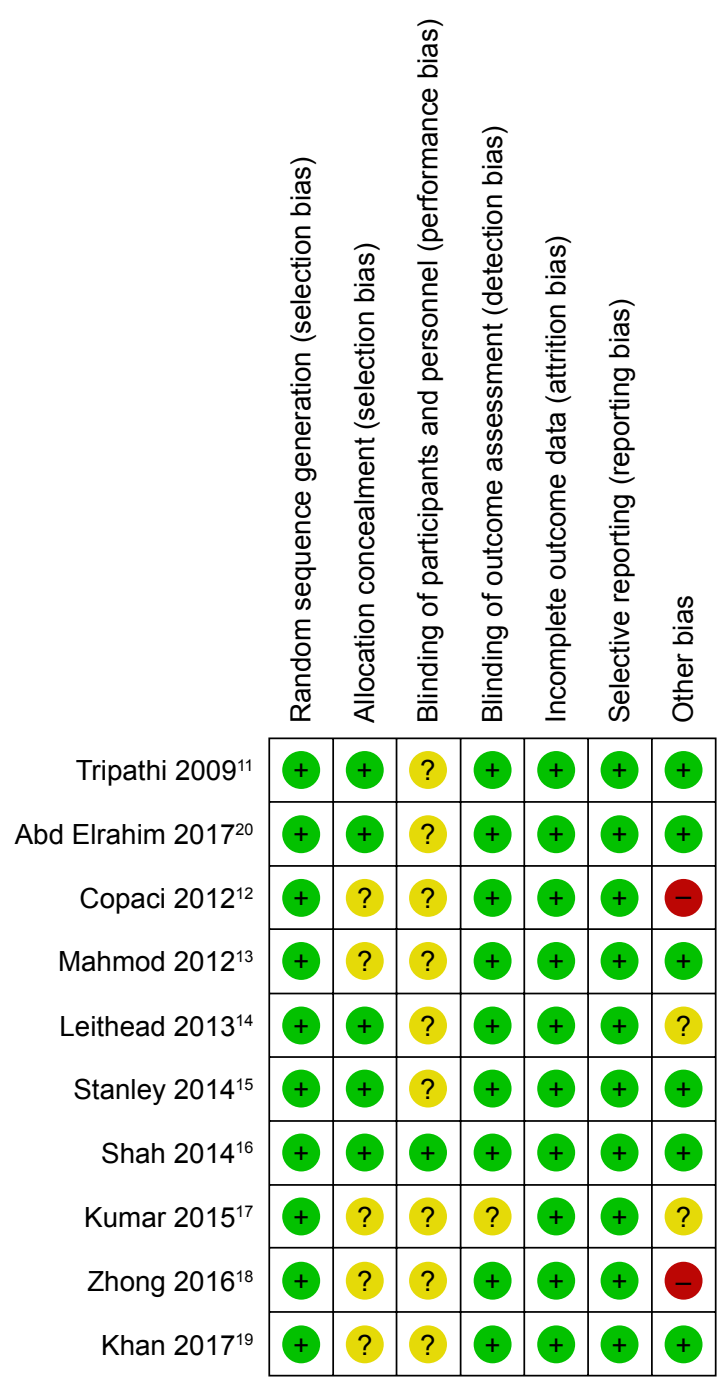

Figure 2 Risk of bias summary of the selected RCTs by the Cochrane tool. Abbreviation: RCTs, randomized controlled trials.

that no significant difference was found in bleeding-related mortality between the two groups (Figure 4).

\section{Overall mortality}

Five trials reported the data of overall mortality. Similarly, subgroup analysis according to outcome measured time was performed. Meta-analyses result in random effects model showed that the pooled RR of overall mortality were 1.11 in 6 months (95\% CI: $\left.0.45-2.73, P=0.82, I^{2}=61 \%\right), 1.22$ in 12 months (95\% CI: $0.78-1.91, P=0.38, I^{2}=0 \%$ ), 0.96 in 18 months (95\% CI: $0.36-2.58, P=0.94, I^{2}=55 \%$ ), and 0.99 in 24 months (95\% CI: $0.53-1.84, P=0.98, I^{2}=64 \%$ ). The results also did not find any significant difference in overall mortality between carvedilol and VBL group (Figure 5).

\section{Other causes of mortality}

Besides bleeding, we further compared the other causes of mortality through subgroup analysis. As summarized in
Table 2, meta-analysis results showed that no significant differences were found in causes of infection $(R R=1.24$, 95\% CI: 0.50-3.10), decompensated liver disease ( $R R=0.69$, 95\% CI: 0.40-1.19), respiratory failure $(\mathrm{RR}=0.67,95 \%$ CI: 0.12-3.91), nonvariceal bleeding $(\mathrm{RR}=0.96,95 \% \mathrm{CI}$ : 0.22-4.13), cerebrovascular event $(\mathrm{RR}=0.69,95 \% \mathrm{CI}$ : 0.14-3.41), cardiovascular event ( $R R=1.70,95 \%$ CI: $0.63-$ 4.55), and others ( $\mathrm{RR}=0.14,95 \% \mathrm{CI}$ : 0.02-1.07).

\section{Portal hypertensive gastropathy}

Two trials reported the data of portal hypertensive gastropathy. Meta-analysis results showed that the pooled RR was 0.49 in 6 months (95\% CI: $\left.0.38-0.64, P<0.00001, I^{2}=0 \%\right)$ and 0.35 in 12 months (95\% CI: $0.26-0.47, P<0.00001, I^{2}=24 \%$ ), indicating a significantly lower incidence of portal hypertensive gastropathy with carvedilol than VBL.

\section{Adverse events}

Adverse events were compared by both of their severity and diagnosis, as shown in Table 3. Meta-analysis results showed that no significant differences were found in minor $(\mathrm{RR}=3.76$, 95\% CI: $\left.0.69-20.54, P=0.13, I^{2}=93 \%\right)$, intolerable $(\mathrm{RR}=0.58$, 95\% CI: $0.20-1.70, P=0.32, I^{2}=81 \%$ ) as well as overall (RR=1.43, 95\% CI: $0.72-2.83, P=0.30, I^{2}=85 \%$ ) adverse events (Figure 6). For specific diagnosis, meta-analysis results showed that carvedilol induced significantly higher incidence of dyspnea ( $\mathrm{RR}=34.86,95 \% \mathrm{CI}$ : 4.90-248.07, $\left.P=0.0004, I^{2}=27 \%\right)$, nausea, and vomiting $(\mathrm{RR}=21.52$, 95\% CI: $2.97-155.92, P=0.002, I^{2}=9 \%$ ), while significantly decreased incidence of transient dysphagia $(R R=0.02,95 \%$ CI: $0.00-0.12, P=0.0001, I^{2}=48 \%$ ). No significant difference in symptomatic hypotension (4/0), bleeding from postbanding ulcer $(0 / 4)$, intolerable banding $(0 / 5)$, and others $(3 / 8)$ was found between the two groups (Table 3 ).

\section{Combination of carvedilol $+\mathrm{VBL}$ versus VBL}

Two trials compared combination therapy of carvedilol and VBL with VBL alone. The meta-analysis results showed that no significant differences were found in variceal bleeding rate $\left(\mathrm{RR}=0.71,95 \% \mathrm{CI}: 0.15-3.30, P=0.67, I^{2}=68 \%\right)$.

\section{Discussion}

\section{Summary of evidence}

The present meta-analysis suggests that carvedilol is equal to VBL for clinical outcomes of cirrhotic portal hypertension, in the available RCTs presented as overall efficacy estimates including both risks of variceal bleeding, mortality, and adverse events. Combined data indicate that the 


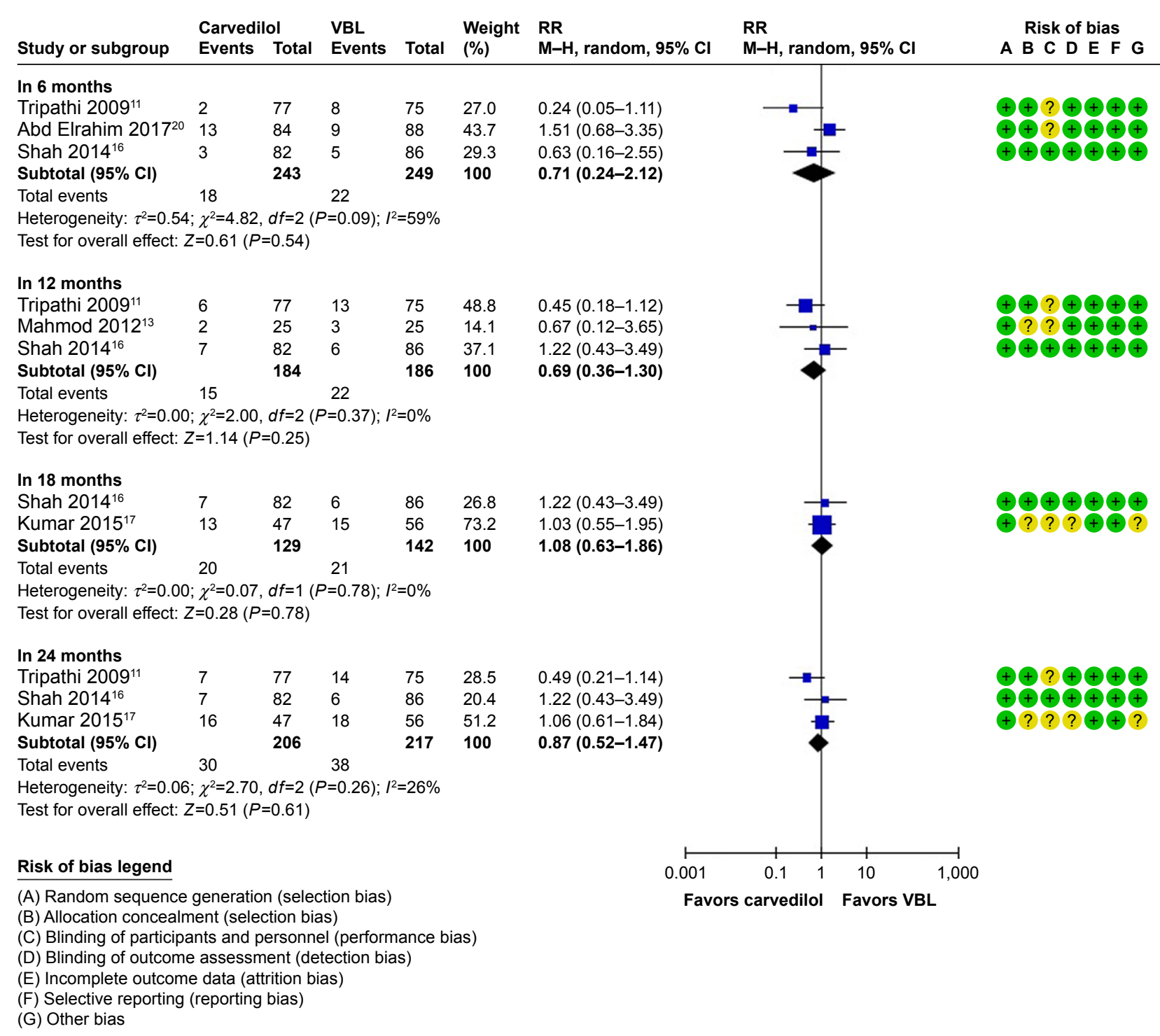

Figure 3 RR (random effects model) of bleeding incidence between carvedilol and VBL in the subgroups of RCTs assessing the preventive efficacy in 6, I2, I8, and 24 months. Abbreviations: RCTs, randomized control trials; RR, risk ratio; VBL, variceal banding ligation.

risks of main outcomes including bleeding and mortality were gradually increased along with follow-up periods from 6 to 24 months after randomization, while no significant difference was found. As the majority of the included patients were aiming at preventing the first episode of bleeding and long-term mortality, our results may be primarily applicable for such a population.

Patients in the two treatment groups were fairly comparable in each study regarding cirrhotic etiology and severity mainly in Child-Pugh scores and Child grade. For HVPG, none of them measured the baseline and lowering effects of carvedilol during follow-up. Although HVPG is recognized to be useful in both judging the hemodynamic response to medical therapy and risk of stratification. ${ }^{23,24}$ However, many studies argue its necessity in carvedilol therapy. ${ }^{11,15,25}$ First, both high-quality studies and meta-analysis demonstrate its high response rate of over $60 \%$ under a wide adopted definition of HVPG $\leq 12 \mathrm{mmHg}$ or by $\geq 20 \%$ reduction of baseline; ${ }^{5,8}$ second, as an invasive and relative complex technique, its accuracy varied in different centers. ${ }^{26}$ Therefore, this situation should not be excessively cautioned.

Regarding the evaluation of variceal bleeding risk between carvedilol and VBL, besides the influence of possible nonresponders in carvedilol group, another key problem is varices eradication that the reported eradication rate in the included four trials were different as $58 \%, 56 \%$, $75 \%$, and $75 \%$, respectively. ${ }^{11,13,16,20}$ The corresponding bleeding risks in VBL group were 17.33\%, 12\%, 6.98\%, and $10.23 \%$. Thus, it seems that a study with a high rate of eradication partly indicates a low risk of post-VBL bleeding. ${ }^{27}$ 


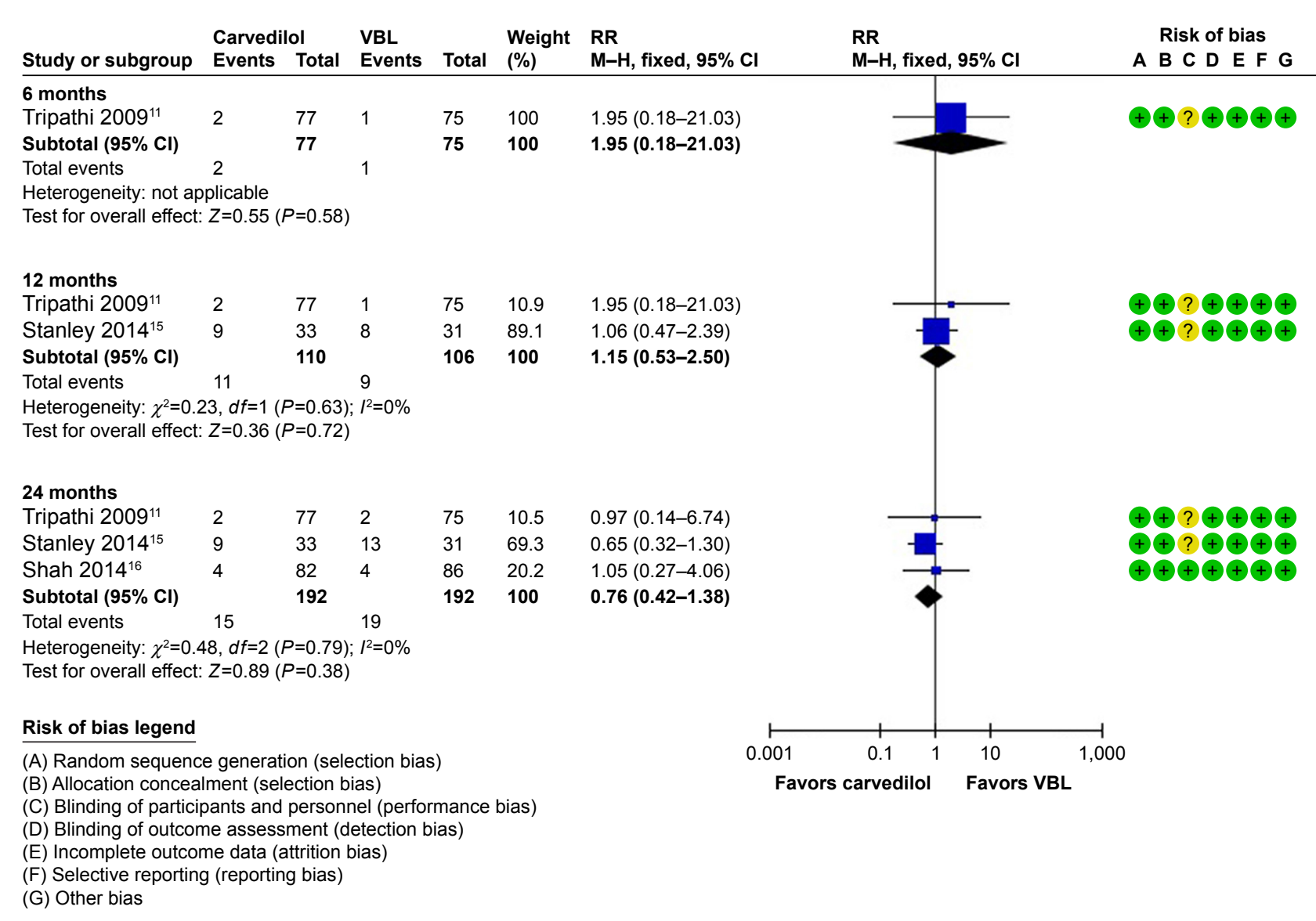

Figure 4 RR (random effects model) of bleeding-related mortality between carvedilol and VBL in the subgroups of RCTs assessing the preventive efficacy in 6, I2, I8, and 24 months. Abbreviations: RCTs, randomized control trials; RR, risk ratio; VBL, variceal banding ligation.

This will be very helpful to explain the inconsistent results of bleeding preventive efficacy across the trials.

Due to the close relationship between clinical prognosis and $\mathrm{HVPG},{ }^{9,28}$ a study reports that portal pressure-guided therapy significantly improves survival in cirrhosis not only because of a great reduction in the risk of rebleeding, but also a reduction in the risk of further decompensation of cirrhosis. ${ }^{29}$ However, in current study, carvedilol as a lowering hepatic venous pressure agent compared with VBL did not obtain an improvement in both bleeding and mortality. For systematic reviews and meta-analyses investigated on pharmacological therapy and endoscopic ligation, 5,30,31 almost consistent results of no significant difference on mortality between the groups were reported, except for Li et al. ${ }^{6} \mathrm{By}$ reviewing the different study, we highly surmised that the result would be obviously affected by their included study of Lo et al, ${ }^{32}$ in which the researcher adopted a combination of nadolol plus isosorbide-5-mononitrate with an average follow-up of 8 years. Additional vasodilator such as isosorbide mononitrate can rescue most of nonresponders of
NSBBs, since separate NSBBs may increase portocollateral resistance. ${ }^{33}$ Taking together, we conclude the possible reasons for the nonsignificant difference of carvedilol compared with VBL for portal hypertension: 1) insufficient reduction in average HVPG (mainly due to nonresponders in monotherapy) and 2) the insufficient follow-up periods, although our study assessed the trend over time and presented the longest follow-up results in 24 months, it was still insufficient under the situation of insufficient reduction in HVPG. A complete success of reduction in HVPG may contribute to a reduction in required follow-up periods. Improved mortality is achieved in the average follow-up of 24 months when applying repeated HVPG measurement to confirm the success of HVPG reduction in a previous study. ${ }^{29}$ Under this hypothesis, further studying a combination of pharmacological therapy appears to be more suitable for the prognosis analysis. Currently, ascites presence and response to NSBBs seem to be more easily realized to predict prognosis in cirrhosis, ${ }^{34,35}$ which may be related to both carvedilol dose and decompensated cirrhosis. 


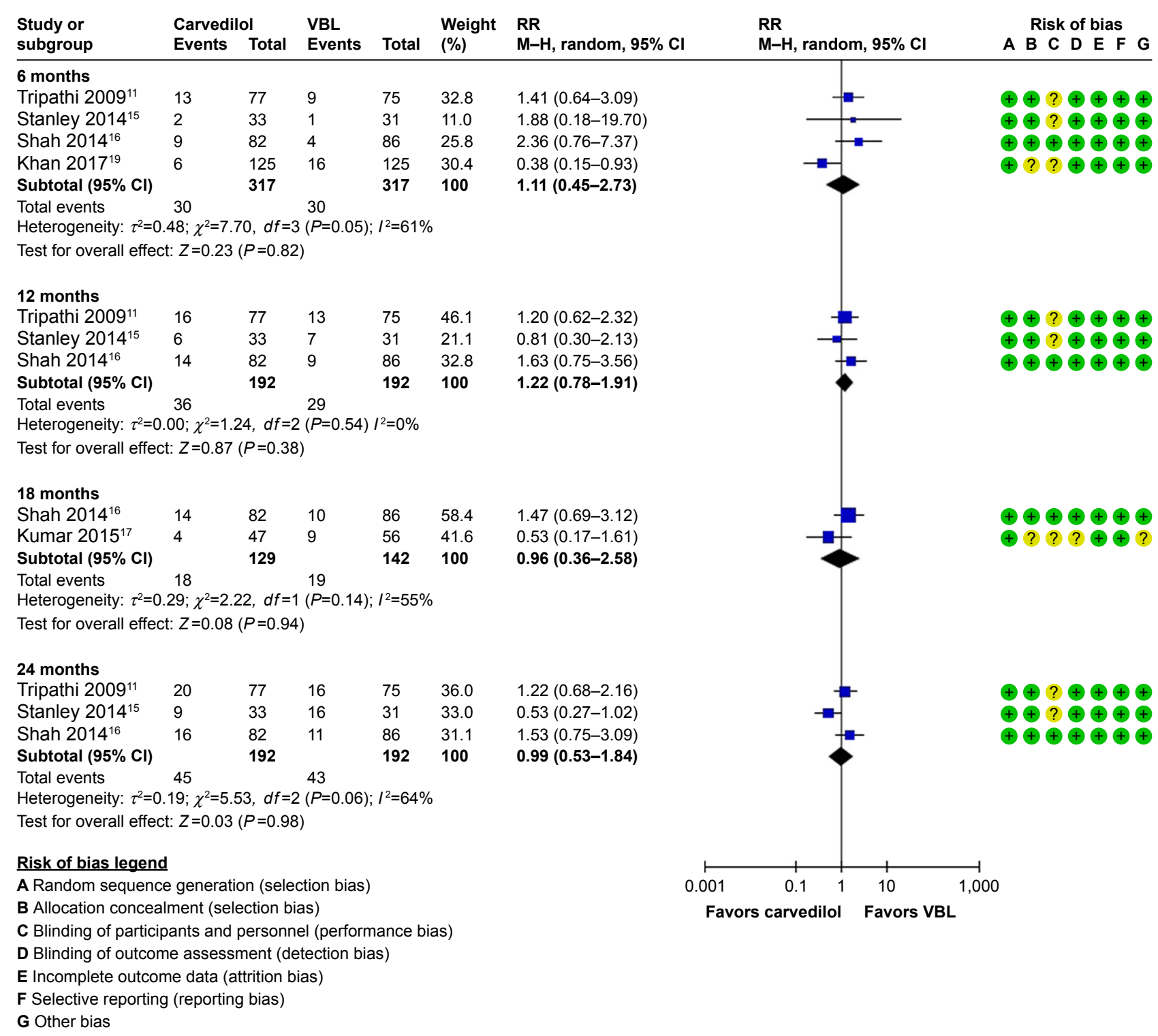

Figure 5 RR (fixed effects model) of overall mortality between carvedilol and VBL in the subgroups of RCTs assessing the preventive efficacy in 6, I2, and 24 months. Abbreviations: RCTs, randomized control trials; RR, risk ratio; VBL, variceal banding ligation.

Table 2 Other causes of mortality in the included RCTs

\begin{tabular}{|c|c|c|c|c|c|}
\hline \multirow[t]{2}{*}{ Diagnosis } & \multirow[t]{2}{*}{ Trials } & \multicolumn{2}{|c|}{ Heterogeneity } & \multicolumn{2}{|l|}{ Effect size } \\
\hline & & $I^{2}(\%)$ & $P$-value & RR (95\% Cl) & $P$-value \\
\hline Infection & I, 5, 6 & 0 & 0.47 & $1.24(0.50-3.10)$ & 0.64 \\
\hline Decompensated liver disease & $I, 5,6$ & 44 & 0.17 & $0.69(0.40-1.19)$ & 0.18 \\
\hline Respiratory failure & 1,6 & 35 & 0.21 & $0.67(0.12-3.91)$ & 0.66 \\
\hline Nonvariceal bleeding & 1,5 & 52 & 0.15 & $0.96(0.22-4.13)$ & 0.96 \\
\hline Cerebrovascular event & 1,5 & 0 & 0.55 & $0.69(0.14-3.41)$ & 0.65 \\
\hline Cardiovascular event & $I, 5,6$ & 0 & 0.45 & $1.70(0.63-4.55)$ & 0.29 \\
\hline Others & I, 5 & 0 & 0.53 & $0.14(0.02-1.07)$ & 0.06 \\
\hline
\end{tabular}

Abbreviations: RCTs, randomized controlled trials; RR, risk ratio. 
Table 3 Adverse events of the included RCTs comparing carvedilol with VBL

\begin{tabular}{|c|c|c|c|c|c|}
\hline \multirow[t]{2}{*}{ Diagnosis } & \multirow[t]{2}{*}{ Trials } & \multicolumn{2}{|c|}{ Heterogeneity } & \multicolumn{2}{|l|}{ Effect size } \\
\hline & & $I^{2}(\%)$ & $P$-value & RR (95\% Cl) & $P$-value \\
\hline Dyspnea & 1,6 & 27 & 0.24 & $34.86(4.90-248.07)$ & 0.0004 \\
\hline Nausea and vomiting & 1,6 & 9 & 0.29 & $21.52(2.97-155.92)$ & 0.002 \\
\hline Transient dysphagia & 10,6 & 48 & 0.17 & $0.02(0.00-0.12)$ & 0.0001 \\
\hline Symptomatic hypotension & 1,6 & 0 & 0.97 & $5.05(0.60-42.76)$ & 0.14 \\
\hline Bleeding from postbanding ulcer & 5,6 & 0 & 0.66 & $0.20(0.02-1.63)$ & 0.13 \\
\hline Intolerable of banding & 1,10 & 0 & 0.64 & $0.17(0.02-1.41)$ & 0.10 \\
\hline Other & 1 & 81 & 0.02 & $0.43(0.13-1.40)$ & 0.16 \\
\hline
\end{tabular}

Abbreviations: RCTs, randomized controlled trials; VBL, variceal banding ligation.

\section{Strengths and limitations}

Important strengths of current systematic review and meta-analysis included the use of rigorous methodology developed by the Cochrane Collaboration and the use of presentation guided by the PRISMA guidelines. Because comprehensive inclusion of available trials is the first step of a well-conducted systematic review and meta-analysis, we employed many methods to control the bias from study selection (publication bias) through literature search of both published or not published studies, and full-texts or meeting abstracts. Since, the quality of the included trials determines the level of evidence of a systematic review and metaanalysis, we assessed methodological quality not only in overall, but also in the outcome analyses, indicating the importance of quality on a reliable and stable outcome. However, one major limitation is that the HVPG reduction was not measured in the included trials, thus a potential advantage of carvedilol on long-term prognosis in portal

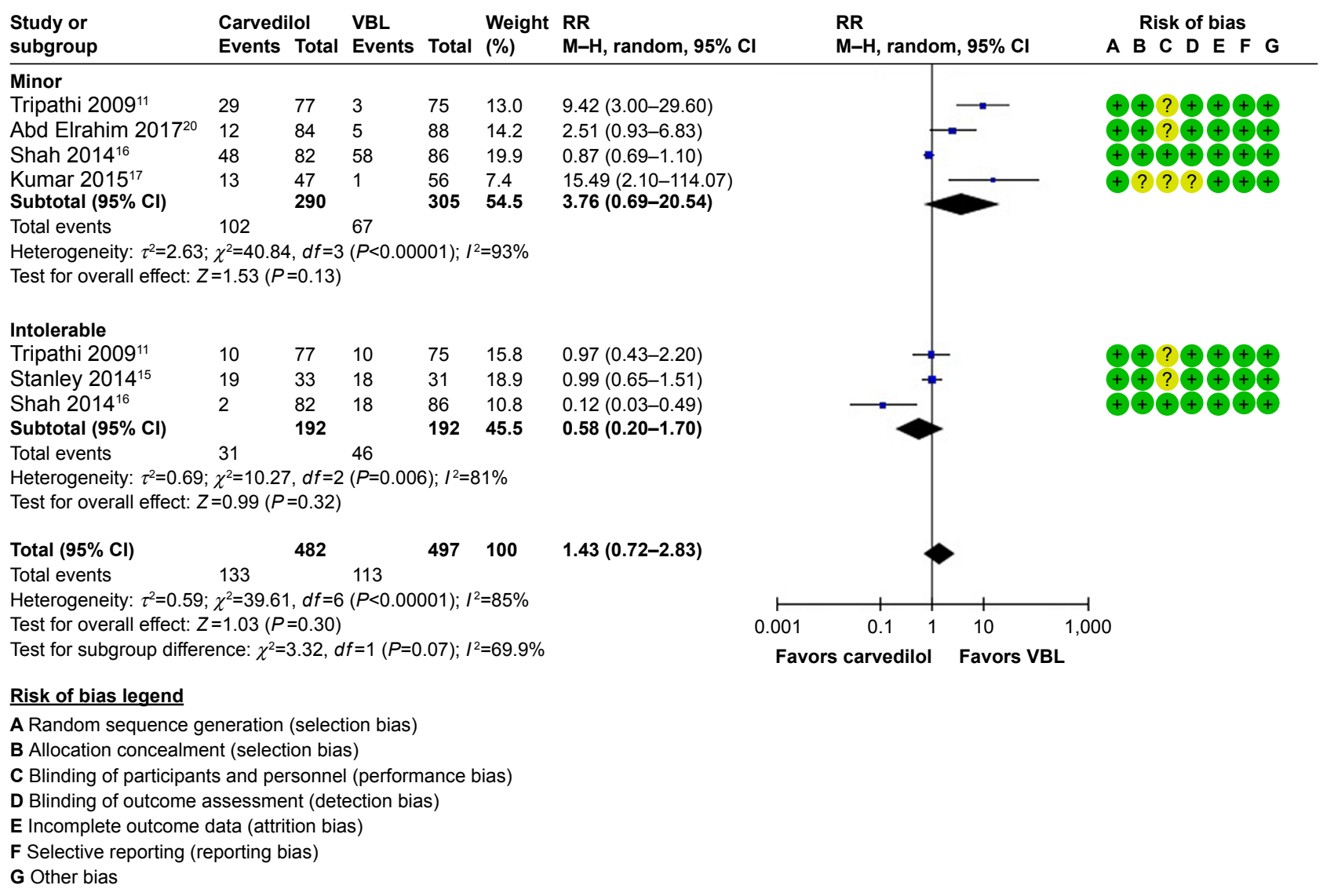

Figure 6 RR (random effects model) of adverse events between carvedilol and VBL in the subgroups of RCTs assessing the minor and intolerable events. Abbreviations: RCTs, randomized control trials; RR, risk ratio; VBL, variceal banding ligation. 
hypertension patients, compared with VBL, is highly suspended to be influenced by the nonresponders and also some noncompliant patients. The other limitation is that the eradication rate varied across the trials; although no confirmed relationship between eradication and bleeding was reported, this may also be a supposed factor introducing heterogeneity.

\section{Agreement and disagreement with other studies or reviews}

In regard to the main outcome measurements of variceal bleeding, bleeding-related mortality and overall mortality, a significant reduction of overall mortality was reported in the meta-analysis of $\mathrm{Li}$ et $\mathrm{al} ;{ }^{6}$ however, they pooled data on various NSBBs and additional vasodilators. First, this may result in misleading conclusions and may be little practical; second, the result was obviously affected by an included study accounted a large weight of $32.8 \%$ and reported a boundary point of 1.01 in the estimate of $95 \%$ CI. The results of our review were consistent with a previous review by Zheng et al. ${ }^{5}$ However, the major questions in regard to their meta-analysis are whether it was appropriate to pool data on four different comparisons (propranolol, VBL, nadolol plus isosorbide-5-mononitrate, and nebivolol) and whether it was reliable to draw a conclusion on the topic of carvedilol vs VBL based on a subgroup analysis including only two trials. In the last fewer years, a number of new relevant studies have been published, our findings add to an accumulated body of evidence on carvedilol with VBL for portal hypertension separately. Collectively, current data support a nonsuperiority of carvedilol to VBL for the prognosis of cirrhotic portal hypertension.

\section{Implications for practice}

In regard to specific recommendations, one important question remains whether carvedilol or VBL should be recommended for cirrhotic portal hypertension patients. From clinical aspects, it is clear that carvedilol is associated with certain advantages as a noninvasive treatment method, though it requires relatively long-term use with unsatisfied patients' compliance. For specific patients with underlying cerebrovascular and/or cardiovascular diseases, it should be administered cautiously and may be limited. VBL as an invasive therapy, can be completed mostly in 1-3 months with about two to four visits to hospital, and relatively less concerns of patients' physical conditions, except for discomfort in the procedure and post bleeding from ulcer.
Besides, based on our study results, for the prevention of the first episode of variceal bleeding, they result in comparable efficacy. Carvedilol should be administered in the manner of a start dose of $6.25 \mathrm{mg}$ and final dose be increased to $12.5 \mathrm{mg}$ in $2-4$ days, which is demonstrated to be well tolerated for most patients. In case of treatment-related incidence, the baseline and follow-up heart rate and blood pressure need to be monitored, meanwhile the related organs function monitoring should also be done additionally, especially for patients with long-term use, in case of chronic liver dysfunction/failure and renal dysfunction/failure. ${ }^{14,36}$ For significant increased risk of dyspnea, nausea and vomiting, and transient dysphagia, most of them are reported to be mild and tolerable. VBL is recommended to be performed every 2-4 weeks in most included trials until esophageal varices became either grade I or eradication (ensure a high eradication rate as possible), and the first VBL should be started early after visits to avoid an unexpected bleeding between intervals. In the included studies, a trial performed VBL within 2 days reported no incidence of bleeding before the first VBL, while another two trials within 7-21 days reported $3(4 \%)$ and $2(6 \%)$ cases of bleeding. ${ }^{11,15}$

For long-term survival, although current study does not support a advantage of carvedilol as a lowering hepatic venous pressure agent compared with VBL, we conclude two key points helpful in prognosis evaluation in the future: sufficient follow-up period and successful HVPG reduction (HVPG $\leq 12 \mathrm{mmHg}$ or by $\geq 20 \%$ reduction of baseline). Overall survival improvement may be expected with carvedilol responders in 24 months with a sample size of 170 in a previous study; $;{ }^{29}$ however, HVPG-guided therapy cannot be widely recommended ${ }^{37}$ which limits our knowledge on actual HVPG in the population. Additionally, carvedilol achieves a significant improvement in the incidence of portal hypertensive gastropathy in 6 months, thus a combination or separate administration of carvedilol for such patients can be recommended under necessary monitoring.

\section{Conclusion}

Our findings reveal its equivalence of carvedilol with VBL for the prevention of first variceal bleeding in cirrhotic hypertension patients. Besides its advantage in improving portal hypertensive gastropathy, our study does not prove its potential advantage in long-term mortality release as a lowering hepatic venous pressure agent, thus it may be argued that we still need evidence from high-quality studies focused on concluded key points of sufficient follow-up period and successful HVPG reduction. 


\section{Acknowledgments}

The research is supported by Gansu Provincial Natural Science Foundation (no. 1606RJZA152) and Lanzhou University Second Hospital research grant (no. YJZY2013-02).

\section{Disclosure}

The authors report no conflicts of interest in this work.

\section{References}

1. Aguilar-Olivos N, Motola-Kuba M, Candia R, et al. Hemodynamic effect of carvedilol vs. propranolol in cirrhotic patients: systematic review and meta-analysis. Ann Hepatol. 2014;13(4):420-428.

2. Carbonell N, Pauwels A, Serfaty L, Fourdan O, Lévy VG, Poupon R. Improved survival after variceal bleeding in patients with cirrhosis over the past two decades. Hepatology. 2004;40(3):652-659.

3. Garcia-Tsao G, Bosch J. Management of varices and variceal hemorrhage in cirrhosis. $N$ Engl J Med. 2010;362(9):823-832.

4. D'Amico G, Garcia-Tsao G, Pagliaro L. Natural history and prognostic indicators of survival in cirrhosis: a systematic review of 118 studies. $J$ Hepatol. 2006;44(1):217-231.

5. Li T, Ke W, Sun P, et al. Carvedilol for portal hypertension in cirrhosis: systematic review with meta-analysis. BMJ Open. 2016;6(5): e010902.

6. Li L, Yu C, Li Y. Endoscopic band ligation versus pharmacological therapy for variceal bleeding in cirrhosis: a meta-analysis. Can $J$ Gastroenterol. 2011;25(3):147-155.

7. Sarin SK, Valla DC, de Franchis R. Revising consensus in portal hypertension: report of the Baveno $\mathrm{V}$ consensus workshop on methodology of diagnosis and therapy in portal hypertension. $J$ Hepatol. 2011;54(5):1082-1083

8. Sinagra E, Perricone G, D'Amico M, Tinè F, D'Amico G. Systematic review with meta-analysis: the haemodynamic effects of carvedilol compared with propranolol for portal hypertension in cirrhosis. Aliment Pharmacol Ther. 2014;39(6):557-568.

9. Suk KT. Hepatic venous pressure gradient: clinical use in chronic liver disease. Clin Mol Hepatol. 2014;20(1):6-14.

10. Kim TY, Lee JG, Sohn JH, et al. Hepatic venous pressure gradient predicts long-term mortality in patients with decompensated cirrhosis. Yonsei Med J. 2016;57(1):138-145.

11. Tripathi D, Ferguson JW, Kochar N, et al. Randomized controlled trial of carvedilol versus variceal band ligation for the prevention of the first variceal bleed. Hepatology. 2009;50(3):825-833.

12. Copaci I, Micu L, Mindrut E, Voiculescu M. 660 Endoscopic variceal ligation alone versus endoscopic variceal ligation plus carvedilol in variceal bleeding prophylaxis in cirrhotic patients. J Hepatol. 2012; 56(2):S261-S262.

13. Mahmod A, Essa S, Abdel Aal E, Dawod H, Eman A. Evaluation of carvedilol in prevention of first attack of variceal hemorrhage in patients with liver cirrhosis. Afro-Egypt J Infect Endem Dis. 2012; 2(2):87-91.

14. Leithead JA, Ferguson JW, Tripathi D. Implications of long-term carvedilol administration for portal hypertension-related renal dysfunction: follow-up from a randomised controlled trial. Hepatology. 2013;58:855A.

15. Stanley AJ, Dickson S, Hayes PC, et al. Multicentre randomised controlled study comparing carvedilol with variceal band ligation in the prevention of variceal rebleeding. J Hepatol. 2014;61(5):1014-1019.

16. Shah HA, Azam Z, Rauf J, et al. Carvedilol vs. esophageal variceal band ligation in the primary prophylaxis of variceal hemorrhage: a multicentre randomized controlled trial. J Hepatol. 2014;60(4): 757-764.

17. Kumar P, Kumar R, Saxena KN. Secondary prophylaxis of variceal hemorrhage: a comparative study of band ligation, carvedilol and propranolol plus isosorbide mononitrate. Indian $J$ Gastroenterol. 2015;34:A54.
18. Zhong M, Zhang B, Xm L. Significance of NSBB in preventing earlier rehaemorrhagia after endoscopic ligation of the patients with esophageal varices. Pract Pharm Clin Remed. 2016;19(1):113-116.

19. Khan MS, Majeed A, Ghauri F. Comparison of carvedilol and esophageal variceal band ligation for prevention of variceal bleed among cirrhotic patients. Age (Years). 2017;52(14.71):54.07-54.54.

20. Abd Elrahim AY, Fouad R, Khairy M, et al. Efficacy of carvedilol versus propranolol versus variceal band ligation for primary prevention of variceal bleeding. Hepatol Int. 2018;12(1):75-82.

21. Moher D, Shamseer L, Clarke M, et al. Preferred reporting items for systematic review and meta-analysis protocols (PRISMA-P) 2015 statement. Syst Rev. 2015;4(1):1.

22. Higgins JPT, Green S. Cochrane handbook for systematic reviews of interventions version 5.0. 2 [updated September 2009]. The Cochrane Collaboration, 2009. Available from: http://www.cochrane-handbook. org, 2010. Accessed December 10, 2018.

23. Garcia-Tsao G, Abraldes JG, Berzigotti A, Bosch J. Portal hypertensive bleeding in cirrhosis: risk stratification, diagnosis, and management: 2016 practice guidance by the American Association for the study of liver diseases. Hepatology. 2017;65(1):310-335.

24. Berzigotti A, Reig M, Abraldes JG, Bosch J, Bruix J. Portal hypertension and the outcome of surgery for hepatocellular carcinoma in compensated cirrhosis: a systematic review and meta-analysis. Hepatology. 2015;61(2):526-536.

25. Thalheimer U, Bellis L, Puoti C, Burroughs AK. Should we routinely measure portal pressure in patients with cirrhosis, using hepatic venous pressure gradient (HVPG) as a guide for prophylaxis and therapy of bleeding and rebleeding? No. Eur J Intern Med. 2011;22(1):5-7.

26. Silva-Junior G, Baiges A, Turon F, et al. The prognostic value of hepatic venous pressure gradient in patients with cirrhosis is highly dependent on the accuracy of the technique. Hepatology. 2015;62(5):1584-1592.

27. Hwang JH, Shergill AK, Acosta RD, et al. The role of endoscopy in the management of variceal hemorrhage. Gastrointest Endosc. 2014;80(2): 221-227.

28. La Mura V, Nicolini A, Tosetti G, Primignani M. Cirrhosis and portal hypertension: the importance of risk stratification, the role of hepatic venous pressure gradient measurement. World J Hepatol. 2015; $7(4): 688$.

29. Villanueva C, Graupera I, Aracil C, et al. A randomized trial to assess whether portal pressure guided therapy to prevent variceal rebleeding improves survival in cirrhosis. Hepatology. 2017;65(5):1693-1707.

30. Gluud LL, Klingenberg S, Nikolova D, Gluud C. Banding ligation versus beta-blockers as primary prophylaxis in esophageal varices: systematic review of randomized trials. Am J Gastroenterol. 2007; 102(12):2842-2848.

31. Thiele M, Krag A, Rohde U, Gluud LL. Meta-analysis: banding ligation and medical interventions for the prevention of rebleeding from oesophageal varices. Aliment Pharmacol Ther. 2012;35(10):1155-1165.

32. Lo GH, Chen WC, Lin CK, et al. Improved survival in patients receiving medical therapy as compared with banding ligation for the prevention of esophageal variceal rebleeding. Hepatology. 2008;48(2):580-587.

33. Kroeger RJ, Groszmann RJ. Increased portal venous resistance hinders portal pressure reduction during the administration of betaadrenergic blocking agents in a portal hypertensive model. Hepatology. 1985;5(1):97-101.

34. Zipprich A, Garcia-Tsao G, Rogowski S, Fleig WE, Seufferlein T, Dollinger MM. Prognostic indicators of survival in patients with compensated and decompensated cirrhosis. Liver Int. 2012;32(9):1407-1414.

35. Bossen L, Krag A, Vilstrup H, Watson H, Jepsen P. Nonselective $\beta$-blockers do not affect mortality in cirrhosis patients with ascites: post hoc analysis of three randomized controlled trials with 1198 patients. Hepatology. 2016;63(6):1968-1976.

36. Rossello X, Pocock SJ, Julian DG. Long-term use of cardiovascular drugs: challenges for research and for patient care. $\mathrm{J} \mathrm{Am} \mathrm{Coll} \mathrm{Cardiol.}$ 2015;66(11):1273-1285.

37. Angeli P, Bernardi M, Villanueva C, et al. EASL Clinical Practice Guidelines for the management of patients with decompensated cirrhosis. J Hepatol. 2018;69(2):406-460. 
Therapeutics and Clinical Risk Management

Dovepress

\section{Publish your work in this journal}

Therapeutics and Clinical Risk Management is an international, peerreviewed journal of clinical therapeutics and risk management, focusing on concise rapid reporting of clinical studies in all therapeutic areas outcomes, safety, and programs for the effective, safe, and sustained use of medicines. This journal is indexed on PubMed Central, CAS,
EMBase, Scopus and the Elsevier Bibliographic databases. The manuscript management system is completely online and includes a very quick and fair peer-review system, which is all easy to use. Visit http://www.dovepress.com/testimonials.php to read real quotes from published authors.

Submit your manuscript here: http://www.dovepress.com/therapeutics-and-clinical-risk-management-journal 